\title{
Stabilization and passification of uncertain systems via static output feedback
}

\author{
Pavel Pakshin \\ Arzamas Polytechnic Institute of R.E. Alekseev \\ Nizhny Novgorod State Technical University \\ 19, Kalinina Street, Arzamas, 607227, RUSSIA \\ pakshin@apingtu.edu.ru \\ D. Peaucelle \\ LAAS-CNRS ; Université de Toulouse, \\ 7 av. du colonel Roche, F-31077 Toulouse, FRANCE \\ peaucelle@laas.fr
}

February 2, 2009

\begin{abstract}
Control of systems with time-varying dynamical characteristics impose either a conservative timeinvariant control strategy that should hold for all time instants or to adopt some adaptive scheme. One of such, called simple adaptive control, is particularly appropriate for control applications such as flexible aircrafts or other fast varying processes. Indeed, it does not need any real-time estimation of time-vaying parameters but adapts based on the unique sensors measurements. Yet, this control strategy, also called passivity-based adaptive control, needs to be applied for the system to be passifiable. Or at least it needs to exhibit a linear combination of the inputs and outputs such that this property holds. The paper is devoted to the search of such linear combinations with practical efficient algorithms. Results are discussed in detail for an uncertain time-varying aircraft control example.
\end{abstract}

\section{Keywords}

LMI, Robustness, Uncertain systems, Passivity, Static output feedback

\section{Introduction}

For more than thirty years a large number of publications are devoted to simple adaptive control $[8,2,13]$. This control strategy is shown on examples to have important properties in terms of adaptation to varying parameters. Moreover, it has the advantage compared to other adaptive strategies such as gain scheduling not to require the real-time knowledge or estimation of the parameters. But this does not mean that simple adaptive control may be applied whatever the system. One of the main assumption for adaptive control to stabilize the closed-loop system is passification of the system, i.e. existence of a feedback gain that makes the system passive. For that reason this adaptive control strategy is also often called passivity based adaptive control.

Assuming the passification property to hold, recent results have completed the existing robustness experiments and extended the first existing robustness results. In [19, 22] robust stabilization 
analysis is studied with respect to time-varying parameters and results are produced in terms of Linear Matrix Inequalities (LMIs). These may be tested efficiently with existing semi-definite solvers such as [20] and easily coded in Matlab environment using YALMIP [16]. These results probably merit more extensions, but the essential issue is to achieve the passification property assumed in the beginning.

More precisely, the passification property is as follows: there should exist a stabilizing static output feedback and linear combination of the output measurement vector $y$ and the reference signal $w$ (called passive output $z$ ) such that the closed-loop is passive with respect to the couple $w, z$. This property has been for long studied assuming that the passive output is the measured vector $z=y$. In such case the passification property is also called "almost passivity" [3], i.e. passive at the expense of finding a static output-feedback. But having $z=y$ limits the method to square systems (same number of inputs and outputs). Therefore extensions have been proposed [9] to the case when $z=G y$ for a given $G$. Then in $[17,19]$ the issue of robust passivity-based adaptive control was proved to have solutions only if $z=G y+D w$, that is if one allows a feed-though gain in the definition of $z$. That results was largely inspired by the results on "schunting" the original system for obtaining passification property, [13].

But in all these results, the matrix $G$ is assumed to be known, obtained by some physical considerations or by other means. Finding this $G$ matrix happens to be a highly complex problem that is as difficult as finding a stabilizing static output-feedback. As a matter of fact any stabilizing static output-feedback may be used to define a matrix $G$. This is one of the papers contribution. The other contribution is the formulation of an heuristic method for designing a stabilizing static output feedback. This problem has no known solution in the general case and is assumed to be NP-hard $[5,6,21]$. Thus the LMI results that we propose do not claim to be a universal method. Nevertheless, based on a result form [1] for solving Riccati equation with LMI maximization, the heuristic has some interesting properties that seems efficient in practice.

The paper is organized as follows. In the next section the problem to be solved is exposed in details. Then in section III the heuristic for stabilizing static output-feedback design is produced. The following section is devoted to the design of the passifying output. Section V illustrates the results on an academic example. A conclusion ends the paper and opens to future intensive tests of the heuristic on more realistic examples.

\section{Problem statement}

The following class of uncertain linear systems is considered

$$
\dot{x}(t)=A(\delta(t)) x(t)+B(\delta(t)) u(t), \quad y(t)=C x(t)
$$

where $x \in \mathrm{R}^{n}$ is the state vector, $u \in \mathrm{R}^{m}$ is the control input vector, $y \in \mathrm{R}^{p}$ is the measured output vector. $\delta(t) \in \mathrm{R}^{N}$ stands for a vector of $N$ time-varying uncertainties. No assumption is made on their derivatives. The model is assumed of affine parallelotopic type, i.e. variations around a central nominal model defined by matrices $(A, B)$ along axes $\left(A_{i}, B_{i}\right)$ :

$$
A(\delta)=A+\sum_{i=1}^{N} \delta_{i} A_{i} \quad, \quad B(\delta)=B+\sum_{i=1}^{N} \delta_{i} B_{i} .
$$

Each uncertain parameter is assumed to be bounded in an interval including zero

$$
\underline{\delta}_{i} \leq \delta_{i} \leq \bar{\delta}_{i} \quad: \quad \underline{\delta}_{i} \leq 0 \quad, \quad \bar{\delta}_{i} \geq 0 .
$$


The set of uncertainties is the set of all functions with bounded values in the upper defined intervals. It is denoted $\boldsymbol{\Delta}$. We define as well the finite set of extremal values (the vertices):

$$
\boldsymbol{\Delta}_{v}=\left\{\delta=\left(\begin{array}{lll}
\delta_{1} & \ldots & \delta_{N}
\end{array}\right): \delta_{i} \in\left\{\underline{\delta}_{i}, \bar{\delta}_{i}\right\}\right\}
$$

Definition 1 A system with input vector $w$, output vector $z$ and state vector $x$ is said to be globally strictly passive if there nonnegative scalar function $V(x)$ and scalar function $\mu(x)>0$ for $x \neq 0$ such that the following holds

$$
V(x(t)) \leq V\left(x_{0}\right)+\int_{0}^{t}\left[w^{T}(s) z(s)-\mu(x(s))\right] d s,
$$

for all $t>0$ and all initial conditions $x(0)$.

In case of uncertain systems, "robust strict passivity" indicates that the strict passivity property holds for all admissible values of the uncertainty. In the remaining the adjective "globally" is eluded since linear systems are considered.

The goal of this paper is to design a matrix $G$ such that there exists a static output feedback control law of the type

$$
u(t)=w(t)-F y(t)
$$

and a feed-through gain $D(\delta(t))$ that make the following closed-loop system robustly strictly passive

$$
\begin{gathered}
\dot{x}(t)=\underbrace{(A(\delta(t))-B(\delta(t)) F C)}_{A_{c}(\delta(t))} x(t)+B(\delta(t)) w(t) \\
z(t)=G \underbrace{C x(t)}_{y(t)}+D(\delta(t)) w(t)
\end{gathered}
$$

This design problem is related to adaptive control issues exposed in $[18,19]$. If one can find such $G$ matrix for a given set of uncertain parameters $\boldsymbol{\Delta}$, one can expect with techniques exposed in upper cited papers to prove stabilization by simple adaptive control of the linear system and efficient $L_{2}$ gain attenuation for an broader set of uncertainties. The simple adaptive control algorithm is as follows

$$
\begin{aligned}
& u(t)=w(t)-K(t) y(t) \\
& \dot{K}(t)=G y(t) y^{T}(t) \Gamma-\phi(K(t)) \Gamma
\end{aligned}
$$

where $\phi$ is usually a corrective function that prevents the adaptive gain $K(t)$ to grow too large towards unrealistic values and where $\Gamma$ is a parameter that tunes the sizes of attractive domains. The major and essential parameter needed for the adaptive control algorithm is the matrix $G$. It should satisfy the upper defined passifiability. Out goal is to have efficient tools for the design of such matrix.

We shall proceed in two steps to design the matrix $G$. First, recalling that strict passivity implies stability, we shall design a robustly stabilizing feedback gain $F$. Second, for that gain $F$ we shall design the matrix $G$ with an additional criterion that the feed-through matrices $D(\delta)$ to be meaningful in practice need to be as close to zero as possible.

\section{Design of static output-feedback gain}

The considered problem is in the context of time-varying uncertainties without bounds on their derivatives. In such case robust stability can only be proved at the expense of a parameter independent Lyapunov certificate that may be chosen quadratic. This corresponds to the well known "quadratic stability" framework [4]. 
The following theorem gives a parametric description (parametrization) of all stabilizing static output-feedback gains. It is based on LQR concept and extends the results of [10] to the considered class of uncertain systems.

Theorem 1 There exists a gain matrix $F$ such that the system (1) is quadratically stabilizable if and only if there exist matrices $P=P^{T}>\mathbf{0}, Q=Q^{T}>\mathbf{0}, R=R^{T}>0$ and a parameter-dependent $L(\delta)$ such that the following conditions hold for all vertices $\delta \in \boldsymbol{\Delta}_{v}$

$$
\begin{gathered}
F C=R^{-1}\left(B^{T}(\delta) P+L(\delta)\right) \\
A^{T}(\delta) P+P A(\delta)-P B(\delta) R^{-1} B^{T}(\delta) P+Q \\
+L(\delta)^{T} R^{-1} L(\delta) \leq \mathbf{0} .
\end{gathered}
$$

Proof: Let $F$ be a quadratically stabilizing gain. Then according to definition it is equivalent to existence of a positive definite matrix $P=P^{T}>\mathbf{0}$ such that

$$
A_{c}^{T}(\delta) P+P A_{c}(\delta)<\mathbf{0} \quad \forall \delta \in \boldsymbol{\Delta} .
$$

$A_{c}(\delta)$ being linear with respect to $\delta$, this inequality holds for all uncertainties if and only if it holds on the vertices

$$
A_{c}^{T}(\delta) P+P A_{c}(\delta)<\mathbf{0} \quad \forall \delta \in \boldsymbol{\Delta}_{v} .
$$

Since the inequality defines a negative definite constraint on a finite set of values, this is also equivalent to the existence of, possibly small, matrices $Q=Q^{T}>\mathbf{0}$ and $R=R^{T}>\mathbf{0}$ such that

$$
A_{c}^{T}(\delta) P+P A_{c}(\delta)+Q+C^{T} F^{T} R F C \leq \mathbf{0} \quad \forall \delta \in \boldsymbol{\Delta}_{v}
$$

Recall that $A_{c}(\delta)=A(\delta)+B(\delta) F C$ and define $L(\delta)=R F C-B^{T}(\delta) P$. Rearranging (12) one gets exactly (9).

The heuristic that is considered in the paper is to replace the non-convex inequality of (9) by the following two constraints

$$
\begin{gathered}
A^{T}(\delta) P+P A(\delta)-P B(\delta) R^{-1} B^{T}(\delta) P+(1+\mu) Q \geq \mathbf{0} \\
\mu Q \geq L^{T}(\delta) R^{-1} L(\delta)
\end{gathered}
$$

where $\mu$ is some positive scalar. A positive scalar $\mu$ such that these conditions (13) hold necessarily exists since $Q$ is positive definite. Moreover, conditions (13) are convex with respect to the decision variables and can be replaced by

$$
\begin{aligned}
& {\left[\begin{array}{cc}
A^{T}(\delta) P+P A(\delta)+(1+\mu) Q & P B(\delta) \\
B^{T}(\delta) P & R
\end{array}\right] \geq \mathbf{0}} \\
& {\left[\begin{array}{cc}
\mu Q & L(\delta) \\
L(\delta)^{T} & R
\end{array}\right] \geq \mathbf{0}}
\end{aligned}
$$

But, solving inequalities (14) may not lead to a solution to the problem (9). To approach such solution, the idea is to attempt to find among solutions of (14) those that make the first inequality of (13) as close as possible to the Riccati equation

$$
A^{T}(\delta) P+P A(\delta)-P B(\delta) R^{-1} B^{T}(\delta) P+(1+\mu) Q=\mathbf{0} .
$$

Indeed, if the Riccati equation holds along with $\mu Q \geq L^{T}(\delta) R^{-1} L(\delta)$ then (9) is valid. The heuristic used to find among solutions the one that may be as close as possible to a solution to the Riccati equation is based on the following theorem borrowed from [1] (only the deterministic result is presented). 
Theorem 2 If the pairs $(A(\delta), B(\delta))$ are stabilizable for all values in the finite set $\Delta \in \boldsymbol{\Delta}_{v}$ and if $(Q(\delta), R(\delta))$ are couples of given positive definite matrices, then the solution to the following LMI maximization problem

$$
\begin{aligned}
& \left\{P^{*}(\delta)\right\}_{\delta \in \boldsymbol{\Delta}_{v}}=\arg \max \sum_{\delta \in \boldsymbol{\Delta}_{v}} \operatorname{Trace}(P(\delta)) \\
& \forall \delta \in \boldsymbol{\Delta}_{v} \\
& P(\delta)=P(\delta)^{T} \\
& {\left[\begin{array}{cc}
A^{T}(\delta) P(\delta)+P(\delta) A(\delta)+\hat{Q}(\delta) & P(\delta) B(\delta) \\
B^{T}(\delta) P(\delta) & R(\delta)
\end{array}\right] \geq \mathbf{0}}
\end{aligned}
$$

are positive semi-definite $P^{*}(\delta) \geq 0$ and solution to the Riccati equations

$$
\begin{aligned}
& A^{T}(\delta) P^{*}(\delta)+P^{*}(\delta) A(\delta) \\
& -P^{*}(\delta) B(\delta) R^{-1}(\delta) B^{T}(\delta) P^{*}(\delta)+\hat{Q}(\delta)=\mathbf{0} .
\end{aligned}
$$

What Theorem 2 suggests is that solving the LMI optimization with maximization of trace of $P$ pushes the solutions to be close to solving the Riccati equation. The result happens to be exact in case one Lyapunov matrix is searched for each Riccati equation. It is assumed to keep the behavior if the variables $P(\delta)$ are constrained, for example if constrained to be all equal $(P(\delta)=P$, $\left.\forall \delta \in \boldsymbol{\Delta}_{v}\right)$.

At this stage the results suggest that performing the maximization of trace of $P$ under constraints (8) and (14) may yield a matrix $F$ robustly stabilizing the uncertain system. Remains to choose appropriately the matrices $Q, R$.

The choice of $Q$ and $R$ is done noticing that these matrices define a linear-quadratic (LQ) problem. Indeed, if $P, \hat{Q}=(1+\mu) Q$ and $R$ are solutions to the following Riccati equation

$$
A^{T} P+P A-P B R^{-1} B^{T} P+\hat{Q}=\mathbf{0}
$$

(which is the goal of the LMI maximization) then $R^{-1} B^{T} P$ is the stabilizing state-feedback gain for the nominal model $\dot{x}=A x+B u$ that minimizes the quadratic cost $\int_{0}^{\infty} x^{T} \hat{Q} x+u^{T} R u d t$. Compared to formulas (8) and (9) it suggests that: if the matrices $\hat{Q}$ and $R$ are appropriately chosen for having some properties for state-feedback for the nominal model, then, at the expense of some variations allowed by introduction of the $L(\delta)$ matrix, these same properties may approximately hold for the uncertain closed-loop system. For example, suppose from practical reasons that one aims at having closed-loop lopes of the system located near to some prescribed values $\lambda_{1}, \ldots \lambda_{n}$. Then Johnson's algorithm [12] allows to compute the $\hat{Q}$ and $R$ matrices such that the optimal state-feedback controller for that LQ problem locates exactly the poles at that prescribed values. Solving the maximization of trace of $P$ under constraints (8) and (14) for the pair $\left(Q=\frac{1}{1+\mu} \hat{Q}, R\right)$ is expected to produce a static-output feedback which locates the closed-loop poles "close" to $\lambda_{1}, \ldots \lambda_{n}$.

Based on the upper considerations, the following heuristic algorithm is proposed for robust stabilizing static output-feedback design

\section{Algorithm 1}

1- Choose a set of desired closed-loop poles $\lambda=\left\{\lambda_{1}, \ldots \lambda_{n}\right\}$ based on practical considerations.

2- For this choice of $\lambda$ apply Johnson's algorithm [12] and get matrices $(\hat{Q}, R)$. 
3- For this choice of $(\hat{Q}, R)$ set $\mu$ to some positive value, take $Q=\frac{1}{1+\mu} \hat{Q}$ and solve the following maximization problem with some LMI solver

$$
\max _{F, P, L(\delta)} \operatorname{trace}(P) \quad: \quad(8),(14) \quad \forall \delta \in \boldsymbol{\Delta}_{v}
$$

The efficiency of the algorithm and its sensitivity to the choices of $\lambda$ and $\mu$ are commented on an example in section $\mathrm{V}$.

\section{Design of $G$ matrix}

Let us now consider the design of a $G$ matrix based on this stabilizing static output feedback gain. For that the results in [19] are recalled with the choice to limit the formula to a unique Lyapunov matrix over all uncertainties (for the same reasons as upper). The theorem is essentially a reformulation of the positive-real lemma [7] and also an LMI version of the SPR conditions recalled in [11].

Theorem 3 The closed-loop system (6) with $D(\delta)=D+\sum_{i=1}^{N} \delta_{i} D_{i}$ is quadratically strictly passive if and only if there exist a symmetric positive-definite matrix $H>\mathbf{0}$ and a positive scalar $\epsilon>0$ such that the following LMI conditions hold for all vertices $\delta \in \boldsymbol{\Delta}_{v}$

$$
\begin{gathered}
{\left[\begin{array}{cc}
A_{c}^{T}(\delta) H+H A_{c}(\delta) & H B(\delta) \\
B^{T}(\delta) H & \mathbf{0}
\end{array}\right]} \\
\leq\left[\begin{array}{cc}
-\epsilon \mathbf{1} & C^{T} G^{T} \\
G C & D(\delta)+D^{T}(\delta)
\end{array}\right]
\end{gathered}
$$

To understand the role of the $D(\delta)$ matrix, assume it is equal to zero. In such case the constraints (15) write also as follows

$$
A_{c}^{T}(\delta) H+H A_{c}(\delta) \leq-\epsilon \mathbf{1}, H B(\delta)=C^{T} G^{T}
$$

which are clearly extremely severe (equality constraints on $P$ ) and almost impossible to attain when $B(\delta)$ is uncertain. If $D(\delta)=\mathbf{0}$ results in [8] also indicate that conditions may be possible if and only if the open loop system defined by $(A(\delta), B(\delta), C)$ is strictly hyper minimum phase (relative degree 1 between numerator and denominator and only stable zeros if system is SISO). This may not be the case for the open-loop system, possibly not even for one value of the uncertainty.

Thus the degree of freedom brought by the feed-through matrix $D(\delta)$ are absolutely needed for solving the problem. But, keeping in mind that passivity properties of a system may be useful in practical control strategies, one may ask for having $D(\delta)$ matrix as close to zero as possible. This expression should be understood in the sense that the norm of the $D(\delta)$ matrix should be as small as possible compared to that of the $G$ matrix. Since bounding from below the norm of $G$ is not convex but keeping in mind that the LMIs (15) are linear with respect to all decision variables (no constant term), a way to handle the minimization of $D(\delta)$ while constraining the norm of $G$ is as follows

$$
\mathbf{1} \leq H \quad \min _{H, G, D(\delta)} \sum_{i=1}^{N} \operatorname{trace}\left(D_{i}\right)
$$

This is why the following algorithm is proposed to solve the $G$-design problem (based on a fixed static output feedback gain $F$ ): 
Algorithm 2 For a given F solve the following minimization problem with some LMI solver

$$
\min _{H, G, D(\delta)} \sum_{i=1}^{N} \operatorname{trace}\left(D_{i}\right) \quad: \quad \mathbf{1} \leq H \quad, \quad(15) \quad \forall \delta \in \boldsymbol{\Delta}_{v}
$$

If $G$ is required to have a norm of $\gamma$, then append the obtained matrices as

$$
G \leftarrow \frac{\gamma}{\|G\|} G, \quad D \leftarrow \frac{\gamma}{\|G\|} D, \quad D_{i} \leftarrow \frac{\gamma}{\|G\|} D_{i}
$$

Proposition 1 The optimization problem of Algorithm 2 is feasible if and only if $F$ is a quadratically stabilizing static output feedback gain.

Proof: $F$ is a quadratically stabilizing static output feedback gain if and only if the exists a positive definite $H>\mathbf{0}$ such that

$$
A_{c}^{T}(\delta) H+H A_{c}(\delta)<\mathbf{0} \quad \forall \delta \in \boldsymbol{\Delta}_{v} .
$$

Since the inequality defines a negative definite constraint on a finite set of values, this is also equivalent to the existence of an $\epsilon>0$ and a, possibly small, matrices $T(\delta)>\mathbf{0}$ such that

$$
\begin{aligned}
& A_{c}^{T}(\delta) P+P A_{c}(\delta)+\epsilon \mathbf{1} \\
& +\left(H B(\delta)-C^{T} G^{T}\right) T(\delta)\left(B^{T}(\delta) H-G C\right) \leq \mathbf{0} \quad \forall \delta \in \boldsymbol{\Delta}_{v}
\end{aligned}
$$

Take $D(\delta)=\frac{1}{2} T^{-1}(\delta)$, a Schur complement argument completes the proof.

\section{$5 \quad$ Examples}

Consider the problem of stabilization of the angular longitudinal aircraft motion under given flight parameters uncertainty. The linearized model of this motion is given by the following equations:

$$
\begin{aligned}
\dot{\vartheta} & =\omega_{z} \\
\dot{\omega}_{z} & =-a_{m z}^{\alpha} \vartheta-a_{m z}^{\omega z} \omega_{z}+a_{m z}^{\alpha} \Theta+a_{m z}^{\delta} \delta \\
\dot{\Theta} & =-a_{y}^{\alpha} \vartheta+a_{y}^{\alpha} \Theta
\end{aligned}
$$

where $\vartheta$ is the pitch angle, $\omega_{z}$ is the angular velocity, $\Theta=\vartheta-\alpha, \alpha$ is the angle of attack, $\delta$ is the elevator angle. The state and control vectors of the considered system are

$$
x=\left(\begin{array}{c}
\vartheta \\
\omega_{z} \\
\Theta
\end{array}\right) \quad, \quad u=\delta .
$$

Usually only $\vartheta$ and $\omega_{z}$ are available for direct measurement and we have

$$
y=\left(\begin{array}{c}
\vartheta \\
\omega_{z}
\end{array}\right)
$$

In the considered flight mode the aircraft has the following parameters uncertainties:

$$
\begin{gathered}
a_{m z}^{\alpha} \in\left[a_{m z 0}^{\alpha}-\Delta a_{m z}^{\alpha}, a_{m z 0}^{\alpha}+\Delta a_{m z}^{\alpha}\right] \\
a_{y}^{\alpha} \in\left[a_{y 0}^{\alpha}-\Delta a_{y}^{\alpha}, a_{y 0}^{\alpha}+\Delta a_{y}^{\alpha}\right] \\
a_{m z}^{\omega z} \in\left[a_{m z 0}^{\omega z}-\Delta a_{m z}^{\omega z}, a_{m z 0}^{\omega z}+\Delta a_{m z}^{\omega z}\right] \\
a_{m z}^{\delta} \in\left[a_{m z 0}^{\delta}-\Delta a_{m z}^{\delta}, a_{m z 0}^{\delta}+\Delta a_{m z}^{\delta}\right]
\end{gathered}
$$


with

$$
\begin{aligned}
& \Delta a_{m z}^{\alpha}=0.3 a_{m z 0}^{\alpha}, \quad \Delta a_{y}^{\alpha}=0.3 a_{y 0}^{\alpha} \\
& \Delta a_{m z}^{\omega z}=0.3 a_{m z 0}^{\omega z}, \quad \Delta a_{m z}^{\delta}=0.5 a_{m z 0}^{\delta} .
\end{aligned}
$$

i.e. $30 \%$ variation is assumed on coefficients of the state matrix and $50 \%$ variation is assumed on the input gain. The nominal numerical values are the following [14]:

$$
\begin{aligned}
& a_{m z 0}^{\alpha}=4.2, \quad a_{y 0}^{\alpha}=-0.77 \\
& a_{m z 0}^{\omega z}=1.5, \quad a_{m z 0}^{\delta}=-7.4 .
\end{aligned}
$$

Algorithms 1 and 2 are applied to the problem for various values of the tuning parameters.

First the influence of the $\mu$ parameter is studied assuming a given choice of initially prescribed closed-loop poles. This choice is taken to be

$$
\lambda=\left(\begin{array}{lll}
-5 & -5 & -5
\end{array}\right) .
$$

Several values of $\mu$ are tested and for all of these, matrices $F$ (from Algorithm 1 ) and the maximal residual

\begin{tabular}{|c|c|c|c|}
\hline$\mu$ & \multicolumn{2}{|c|}{$F$} & $\mid \mathcal{R} \|$ \\
\hline 0.01 & -0.9456 & -0.0348 & 351.102 \\
\hline 0.1 & -2.4306 & -0.3099 & 348.563 \\
\hline 1 & -4.9398 & -0.9672 & 337.377 \\
\hline 10 & -4.9504 & -0.9701 & 336.607 \\
\hline 100 & -4.9340 & -0.9668 & 336.607 \\
\hline
\end{tabular}

$$
\begin{aligned}
& \|\mathcal{R}\|=\max _{\delta_{\Delta_{v}}} \\
& \quad\left\|A^{T}(\delta) P+P A(\delta)-P B(\delta) R^{-1} B^{T}(\delta) P+(1+\mu) Q\right\|
\end{aligned}
$$

of the Riccati equations are given in Table 1. One can notice that the results for $\mu>1$ are all close to each other and lead to the minimal Riccati residual. This residual is not zero, as expected, yet as seen in the next table the $F$ matrices do stabilize in all cases.

Table 1: $F$ and $\|\mathcal{R}\|$ versus $\mu$

For each of the obtained $F$ matrices, Algorithm 2 is run and prove in all cases the closed-loop robust stability. The obtained $G$ matrices are given along with the obtained maximal value of $D(\delta)$ over all uncertainties, see Table 2 . Note that for the small values of $\mu$ the obtained $G$ matrices are not satisfactory since the feed-through $D(\delta)$ term is dominant compared to the $G$ gain. It is more as if passivity of the trivial feed-though gain is not too much disturbed by the system itself. Such cases are not much useful for passivity based adaptive control and should not be considered.

\begin{tabular}{|c|cc|}
\hline$\mu$ & $G$ & $\max (D(\delta))$ \\
\hline 0.01 & {$\left[\begin{array}{ll}3.8849 & -7.3751\end{array}\right]$} & 12.6840 \\
0.1 & {$\left[\begin{array}{cc}0.8301 & -6.4615\end{array}\right]$} & 1.9299 \\
1 & {$\left[\begin{array}{cc}-0.5016 & -5.9447\end{array}\right]$} & 0.6656 \\
10 & {$\left[\begin{array}{ll}-0.5037 & -5.9437\end{array}\right]$} & 0.6638 \\
100 & {$\left[\begin{array}{ll}-0.5032 & -5.9450\end{array}\right]$} & 0.6659 \\
\hline
\end{tabular}

Table 2: $G$ and $D(\delta)$ versus $\mu$ 
As a second series of test, the influence of $\lambda$ is studied. The parameter $\mu=1$ is kept constant and the same experiments are done. Results of Algorithms 1 and 2 are given in tables 3 and 4 respectively.

\begin{tabular}{|c|c|c|c|c|c|}
\hline \multicolumn{3}{|c|}{$\lambda$} & \multicolumn{2}{|c|}{$F$} & $\|\mathcal{R}\|$ \\
\hline-1 & -1 & -1 & infes & sible & \\
\hline-2 & -2 & -2 & -0.3637 & -0.2549 & 1.95031 \\
\hline-3 & -3 & -3 & -1.3951 & -0.4908 & 16.2933 \\
\hline-5 & -5 & -5 & -4.9398 & -0.9672 & 337.377 \\
\hline-8 & -8 & -8 & -12.8876 & -1.5149 & 6072.42 \\
\hline
\end{tabular}

Table 3: $F$ and $\|\mathcal{R}\|$ versus $\mu$

For the first tested value of $\lambda$ the $F$ design LMIs are found infeasible. Moreover some more tests show that for that $\lambda=\left(\begin{array}{lll}-1 & -1 & -1\end{array}\right)$ the $\mathrm{F}$ design LMIs are never valid. It indicates some sensitivity of the Algorithm to the choice of matrices $Q$ and $R$. The Riccati residual indicates as well that the found feedback gain may have characteristics quite different from those expected when choosing $Q$. Indeed, plots of closed loop poles of Figure 1 show both a tendency of the poles to follow the prescribed $\lambda$, but as this objective become harder to attained with static output-feedback gain, the gap between prescribed and actual closed-loop poles grows.

\begin{tabular}{|c|c|c|c|c|c|}
\hline \multicolumn{3}{|c|}{$\lambda$} & \multicolumn{2}{|c|}{$G$} & $\max (D(\delta))$ \\
\hline-2 & -2 & -2 & -3.680 & -7.3767 & 2.6597 \\
\hline-3 & -3 & -3 & -2.023 & -6.2924 & 1.3026 \\
\hline-5 & -5 & -5 & -0.501 & -5.9447 & 0.6656 \\
\hline-8 & -8 & -8 & 0.937 & -5.8400 & 0.4322 \\
\hline
\end{tabular}

Table 4: $G$ and $D(\delta)$ versus $\mu$

To illustrate the robustness, step responses of the system are plotted in Figure 2. The plotted output is the signal $z=G y+D(\delta) w$ with respect to which the system is passive.

All programming is done in Matlab@environment. LMIs are coded with YALMIP parser [16] and using SeDuMi solver [20]. The computation are done on a Linux PC computer with i686 processor and 2GB memory. Each LMI optimization problem is solved in less than half a second.

\section{Conclusions}

The issue of the design of the passive output vector needed for simple adaptive control has been studied and a heuristic for it has been proposed. This heuristic needs to be validated on realistic examples and this is the goal of ongoing tests. The preliminary tests on the library of control problems of [15] give encouraging results that need to be analyzed in details. The contribution paves as well the way for application of simple adaptive control for many new systems for which the passive output vector could not be easily exhibited. It may be of major importance for systems with rapidly varying parameters such as agile aeronautical systems. 

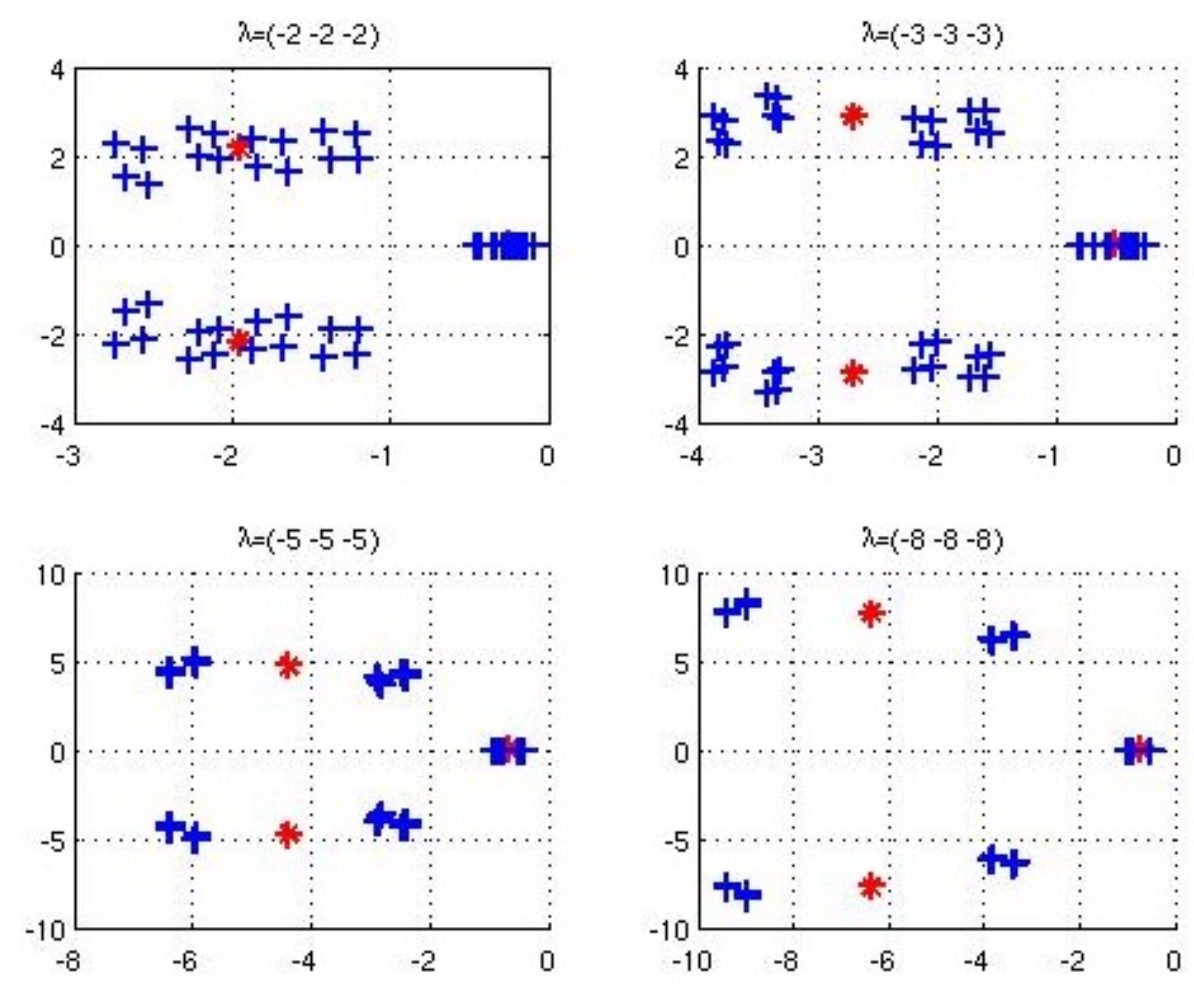

Figure 1: Pole locations for the four control gains of Table 3. Poles for the 16 extremal values of the uncertainty set are denoted by a ' + ' sign, those for the nominal model are denoted by a '*'.

\section{References}

[1] M. Ait Rami and L. El Ghaoui. LMI optimization for nonstandard Riccati equations arising in stochastic control. IEEE Trans. on Automat. Control, 41(11):1666-1671, 1996.

[2] I. Barkana and H. Kaufman. Global stability and performance of an adaptive control algorithm. Int. J. Control, 42(6):1491-1505, 1985.

[3] I. Barkana, M.C.M. Teixeira, and L. Hsu. Mitigation of symmetry condition in positive realness for adaptive control. Automatica, 42(9):1611-1616, September 2006.

[4] B.R. Barmish. Necessary and sufficient conditiond for quadratic stabilizability of an uncertain system. J. Optimization Theory and Applications, 46(4), August 1985.

[5] D. Bernstein. Some open problems in matrix theory arising in linear systems and control. Linear Algebra Applications, 162-164:409-432, 1992.

[6] V. Blondel, M. Gevers, and A. Lindquist. Survey on the state of systems and control. European J. of Control, 1:5-23, 1995.

[7] S. Boyd, L. El Ghaoui, E. Feron, and V. Balakrishnan. Linear Matrix Inequalities in System and Control Theory. SIAM Studies in Applied Mathematics, Philadelphia, 1994.

[8] A.L. Fradkov. Adaptive stabilization of a linear dynamic plant. Autom. Remote Contr., 35(12):1960-1966, 1974. 


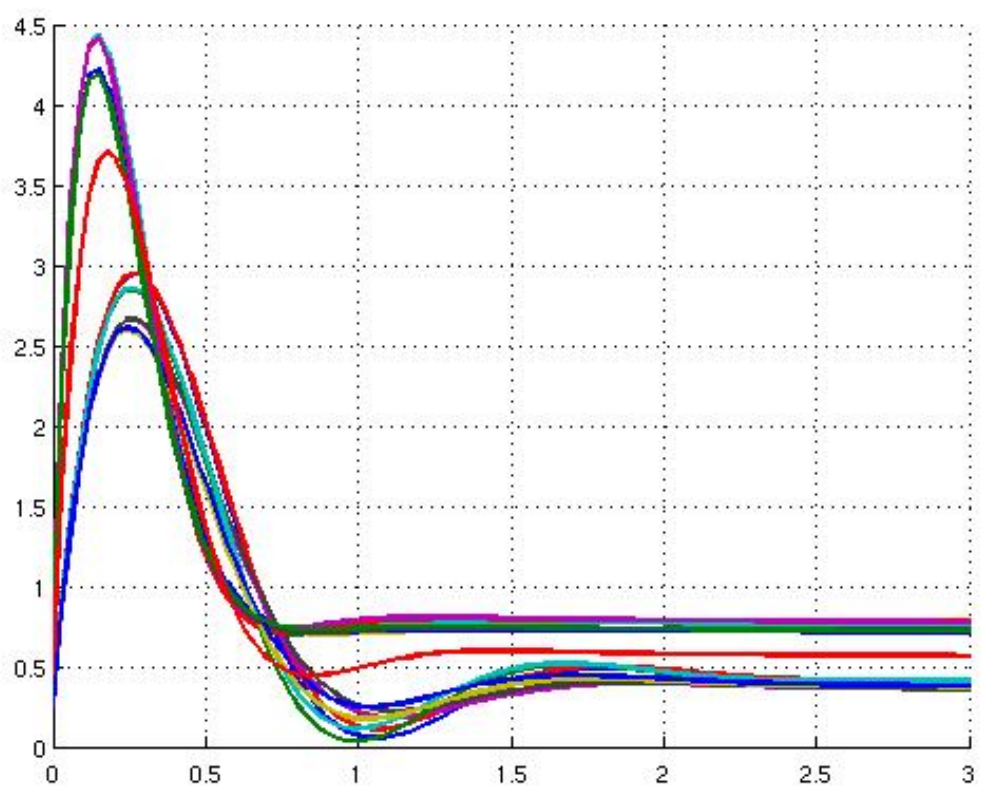

Figure 2: Step responses of closed-loop (extremal and nominal values of uncertainties) in case $\mu=1$ and $\lambda=\left(\begin{array}{lll}-5 & -5-5\end{array}\right)$.

[9] A.L. Fradkov. Passification of non-square linear systems and feedback Yakubovich-KalmanPopov lemma. European J. of Control, 6:573-582, 2003.

[10] J. Gadewadikar, F.L. Lewis, L. Xie, V. Kucera, and M. Abu-Khalaf. Parameterization of all stabilizing $H_{\infty}$ static state-feedback gains: Application to output-feedback design. Automatica, 43:1597-1604, 2007.

[11] P. Ioannou and J. Sun. Robust Adaptive Control. Prentice Hall, Inc, 1996.

[12] C.D. Johnson. The "unreachable poles" defect in LQR theory: analysis and remedy. Int. J. Control, 47:697-709, 1988.

[13] H. Kaufman, I. Barkana, and K. Sobel. Direct adaptive control algorithms. Springer, New York, 1994.

[14] A.A. Krasovskii. Systems of the automatic flight control and their analytical design. Nauka, Moscow, 1973.

[15] F. Leibfritz. COMPleib: COnstraint Matrix-optimization Problem library - a collection of test examples for nonlinear semidefinite programs, control system design and related problems. Technical report, University of Trier, Department of Mathematics, 2004. URL: www. complib.de.

[16] J. Löfberg. YALMIP : A Toolbox for Modeling and Optimization in MATLAB, 2004. URL: http:// control.ee.ethz.ch/ joloef/ yalmip.php. 
[17] D. Peaucelle and A. Fradkov. LMI conditions for robust adaptive control of LTI systems. In IFAC Workshop on Adaptation and Learning in Control and Signal Processing, St. Petersburg, August 2007. Paper in Invited Session "Passification-based Adaptive and Robust Control".

[18] D. Peaucelle, A. Fradkov, and B. Andrievsky. Passification-based adaptive control of linear systems: Robustness issues. Int. J. of Adaptive Control and Signal Processing, 22(6):590-608, August 2008. doi: 10.1002/acs.1009.

[19] D. Peaucelle and A.L. Fradkov. Robust adaptive $L_{2}$-gain control of polytopic MIMO LTI systems - LMI results. Systems $\&$ Control Letters, 57(11):881-887, 2008.

[20] J.F. Sturm. Using SeDuMi 1.02, a MATLAB toolbox for optimization over symmetric cones. Optimization Methods and Software, 11-12:625-653, 1999. URL: http://sedumi.mcmaster.ca/.

[21] V.L. Syrmos, C. Abdallah, and P. Dorato. Static output feedback: A survey. In IEEE Conference on Decision and Control, pages 837-842, December 1994.

[22] I. Yaesh and U. Shaked. Simplified adaptive control via improved robust positive real conditions. Systems \& Control Letters, 55:1033-1040, 2006. 